\title{
Perioperative Nutritional Support or Perioperative Fasting?
}

\section{A narrative review}

\section{Piero Chirletti ${ }^{1}$, Noemi Giannetta ${ }^{2}$, Mariachiara Figura ${ }^{3}$, Angelo Cianciullii ${ }^{4}$, Francesca Malandra ${ }^{4}$, Luca Sacco5 ${ }^{5}$, Sara Dionisi' ${ }^{2}$, Emanuele Di Simone', Marco Di Muzio ${ }^{6}$}

1. MD, Full Professor of Surgery, Head of General and Pancreatic Surgery Unit, Department of Surgical

Sciences, Sapienza University of Rome, Italy.

2. PhD Student, Department of Biomedicine and Prevention, Tor Vergata University of Rome, Italy

3. RN, MSN, Sapienza University of Rome, Rome, Italy

4. RN, Sapienza University of Rome, Rome, Italy.

5. MD, Department of Surgical Sciences, Sapienza University of Rome, Italy

6. PhD, Department of Clinical and Molecular Medicine, Sapienza University of Rome, Italy

* CORRESPONDING AUTHOR: Prof. Piero Chirletti, Department of Surgical Sciences, Sapienza University of

Rome, Viale del Policlinico, 155, 00161 Rome, Italy - piero.chirletti@ uniroma1.it, Tel. +39 0649970385

\begin{abstract}
Traditionally, overnight fasting before elective surgery has been the routine in medical practice for risk reduction of pulmonary aspiration of gastric contents. Several original study and international societies recommend a 2 -h preoperative fast for clear fluids and a 6 -h fast for solids in most elective patients. We conducted a narrative review of the literature, searching electronic databases (Medline and CINAHL). We used PICO approach. The results of our review suggest that nutrition support in the perioperative period is very important to reduce length of hospital stay and reduced postoperative complication.
\end{abstract}

Keywords: ERAS Protocol; nutrition support; fasting; colorectal surgery

\section{Introduction}

Traditionally, overnight fasting before elective surgery has been the routine in medical practice for risk reduction of pulmonary aspiration of gastric contents [1,2]. A period of starvation ("nihl by mouth") is observed after traditional gastrointestinal surgery until intestinal activity has been resumed. But, some studies have shown that prolonged fasting instead causes an increase in patient discomfort and it can result in delayed healing [3, 4].

To reduce surgical patients' length of stay and complication rates, Enhance recovery after surgery (ERAS) Society recommends a multimodal approach. The goals of ERAS protocols include attenuating the surgical stress response and reducing end organ dysfunction through integrated preoperative, intraoperative, and postoperative pathways. Initiated by Professor Henrik Kehlet in Denmark in the 1990s, the ERAS Protocol allows the patient to take nutrients up to a few hours before surgery $[5,6,7,8]$.

In order to the known rapid gastric emptying of clear liquids and the slower digestion and emptying of solids; guidelines recommend patients to freely consume [9, 10, 11]:

- Clear liquids (water, black coffee or tea, fruit juice) until 2 hours before surgery;

- Easily digestible solids until 6-12 hours before surgery;

- Meals with protein content and lipid until 9 hours before surgery;

- Fibers until 9 hours before surgery. 
Nutrition preparation leads to a significant reduction in thirst, hunger and, at the same time, drinking certain beverages provides energy [8].

The aim of this review was therefore critically to appraise the evidence available regarding the pre-operative nutrition for elective colorectal surgery.

\section{Materials and Methods}

\subsection{Selection Criteria}

Original research and review articles on the topic of pre-operative and post-operative nutrition were included in this narrative review. For this purpose, the authors drafted a protocol based on population, intervention, comparison and outcome (PICO) approach.

\begin{tabular}{cr}
\hline P & Patients underwent colorectal surgery for cancer \\
\hline I & Nutritional Support in the Perioperative Period - ERAS Protcol \\
\hline C & Nutritional Support in the Perioperative Period - Traditional approach \\
\hline O & Decreased length of hospital stay - Less incidences of postoperative complications \\
\hline
\end{tabular}

The inclusion criteria for final analysis were as follows:

- Intervention studies including RCTs, Controlled Clinical Trials (CCTs), Cohort analytic, Casecontrol, review, etc;

- Studies reporting the nutritional support in the preoperative period;

- Peer-reviewed research articles published in English and Italian.

The exclusion criteria were: studies reporting educational interventions and grey literature including theses, conference, proceedings, etc.

\subsection{Search Strategy}

Two reviewers searched the bibliographic databased Pubmed and Cinahl for all English and Italian language articles between 2007 and 2017. To obtain an exhaustive string search, through Boolean operators AND and OR, the following keywords were combined: fast track surgery, nutrition, ERAS, ERAS nutrition, nutrition perioperative, enteral nutrition.

\subsection{Procedure}

The initial research's results are:

- PubMed: 40 records;

- CINAHL: 107 records;

Each citation found in the databases was reviewed independently by two authors via a title-first approach to obtain records for the abstract screening, according to an inclusion and exclusion criteria decided beforehand. Each author independently examined the full texts of studies that passed the title and abstract review and met the inclusion criteria. A total of 8 articles were examined.

\section{Results}

The results obtained following our search strategy are included in Table 1. 


\begin{tabular}{|c|c|c|}
\hline Source & Study type & Outcome \\
\hline [11] & & $\begin{array}{l}\text { "The consumption of carbohydrate-loading drink is safely consumed a few hours } \\
\text { before surgery" }\end{array}$ \\
\hline & Original article & $\begin{array}{l}\text { "A slight decrease in the average duration of hospitalization was observed for the } \\
\text { fast-track group }(5.31 \pm 0.98 \text { days }) \text { compared to the conventional group ( } 5.38 \pm \\
2.80 \text { days)". }\end{array}$ \\
\hline & & $\begin{array}{l}\text { "Malnourished patients who receive micronutrient supplementation } \\
\text { preoperatively have lower postoperative inflammatory responses and better } \\
\text { prognoses". }\end{array}$ \\
\hline [14] & Original article & $\begin{array}{l}\text { "Preoperative nutrition risk tended to result in a greater 30-day hospital } \\
\text { readmission rate compared to well-nourished patients" }\end{array}$ \\
\hline [15] & & $\begin{array}{l}\text { "The use of carbohydrate loading attenuates postoperative insulin resistance, } \\
\text { reduces nitrogen and protein losses, preserves skeletal muscle mass and reduces } \\
\text { preoperative thirst, hunger and anxiety. It involves the use of clear carbohydrate } \\
\text { drinks the day prior to surgery and up to } 2 \text { hours before. In addition to the } \\
\text { metabolic effects, it facilitates accelerated recovery through early return of bowel } \\
\text { function and shorter hospital stay, ultimately leading to an improved } \\
\text { perioperative well-being". }\end{array}$ \\
\hline [16] & Original article & $\begin{array}{l}\text { "Despite wide awareness of the 'enhanced recovery after surgery' nutrition } \\
\text { guidelines in those surveyed and attempts to change practice, no hospital in the } \\
\text { study had fully implemented the guidelines". }\end{array}$ \\
\hline [17] & Original article & $\begin{array}{l}\text { "Perioperative continuation of postpyloric EN is feasible in some critically ill } \\
\text { surgical patients and can result in additional calories provided". }\end{array}$ \\
\hline [18] & Original article & $\begin{array}{l}\text { "Postoperative fasting period might have been influenced by the lack of } \\
\text { synchrony between clinical meeting and nutrition and dietetics service schedules. } \\
\text { Besides, lack of knowledge prevented to order a special meal". }\end{array}$ \\
\hline
\end{tabular}

Nutrient delivery in the immediate perioperative period has been the subject of numerous original research and review articles. However, there is no standardized procedure that can cover all of the aims of the nutrition management in perioperative period $[16,18]$.

Several studies show that nutrition preparation leads to a significant reduction in thirst, hunger and, at the same time, drinking certain beverages provides energy [8]. Burch [11] describes how the use of a preoperative carbohydrate-loading drink can be safely consumed a few hours before surgery and it is associated with fewer complications and a shorter length of stay in hospital. This pathway is in accordance with ERAS program. These findings are consistent with the large body of evidence showing that the use of carbohydrate loading reduces preoperative thirst, hunger and anxiety [15].

According to these findings, Kim et al. [12] designed a study to compare two independent samples: patients who were placed in a conventional program and patients who were placed in a fast-track program using the care map. The fast-track concept adopted by authors included also the following steps: no bowel preparation, pre-operative oral hydration, oral intake as soon as possible post-operatively. This led to a slight decrease in the average duration of hospitalization and a decrease of complication rates in fast-tack group. 
Additionally, Liu M-Y [13] adopted malnutrition screening tool (MST) to classified the risk of malnutrition. When a patient was recognized as having high malnutrition risk before surgery, Liu encourage the patient to eat by oral or enteral feeding as tolerated in order to supply nutrition. As preoperative nutritional support, Liu administered a modified form of peripheral parenteral nutrition with added fat emulsion infusion, multiple vitamins, and trace elements. This pathway resulted winner: malnourished patients who receive micronutrient supplementation preoperatively have lower postoperative inflammatory responses and better prognoses.

About nutrition risk and management is the study conducted by Gillis et al [14]. Preoperative nutrition education may be useful to optimize nutrition status [14,12] and, additionally, adequacy of dietary intake was determined for the first 3 postoperative days [14]. So, it is necessary a multidisciplinary approach and an institutional policy to optimize patient's nutrition status $[16,17,18,19]$.

\section{Discussion \& Conclusions}

The results of our review suggest that nutrition support in the perioperative period is very important to reduce length of hospital stay and reduced postoperative complication [20]. Additionally, several original study and international societies recommend a 2-h preoperative fast for clear fluids and a 6-h fast for solids in most elective patients.

Despite our findings, this study has some limitations, mainly due to the fact that it is a narrative review. More original research on the efficacy of nutritional support in the perioperative period is needed before more systematic or meta-analytical reviews can be undertaken. Another limitation may have been introduced by the eligibility criteria employed in the current narrative review. Especially, English language studies were mostly included while original research, published before 2007, were not included. Likewise, grey literature were not included in the review, meaning that further potentially relevant evidence may have been disregarded.

Acknowledgments: This research received no specific grant from any funding agency in the public, commercial, or not-for-profit sectors.

Conflicts of Interest: The authors declare that the research was conducted in the absence of any commercial or financial relationships that could be construed as a potential conflict of interest.

\section{References}

1. Boyle HE, Hewer CL. Practical anaesthetics. 3rd ed. London: Frowde, Hodder and Stoughton; 1923.

2. Pogatschnik C, Steiger E. Review of Preoperative Carbohydrate Loading. NutrClinPract; 2015; 30 (5): 660-664. doi: 10.1177/0884533615594013.

3. Steenhagen E. Enhanced Recovery After Surgery: It's Time to Change Practice! NutrClinPract; 2016; 31(1):18-29. doi: 10.1177 / 0884533615622640.

4. McElroy LM, Codner PA, Brasel KJ. A pilot study to explore the safety of perioperative post pyloric enteral nutrition. Nutr Clin Pract; 2012; 27(6): 777-780.

5. Slater R. Impact of an enhanced recovery programme in colorectal surgery. Br J Nurs; 2010; 19(17):1091-1099. doi: 10,12,968 mila / bjon.2010.19.17.78555.

6. Handley A. Fast Track to Recovery. Nurs Stand; 2009; 24(9): 18-19.

7. Foss M, Bernard H. Enhanced recovery after surgery: implications for nurses. Br J Nurs; 2012; 21(4): 221-223.

8. Horosz B, Nawrocka K, Malec-Milewska M. Anaesthetic perioperative management according to the ERAS protocol. Anaesthesiol Intensive Ther; 2016; 48(1): 49-54. doi: 10.5603 / AIT.2016.0006.

9. Mcleod R, Fitzgerald W, Sarr M. Preoperative fasting guidelines. Canadian Journal of Surgery, 2006, 49.2: 139.

10. American Society of Anesthesiologists Committee, et al. Practice guidelines for preoperative fasting and the use of pharmacologic agents to reduce the risk of pulmonary 
aspiration: application to healthy patients undergoing elective procedures: an updated report by the American Society of Anesthesiologists Committee on Standards and Practice Parameters. Anesthesiology, 2011, 114.3: 495.

11. Burch J. Preoperative carbohydrate loading in the enhanced recovery pathway. Br J Nurs 2016 Jun 23; 25(12):669-672.

12. Kim B, et al. Outcomes of Fast-Track Program after Colorectal Cancer Surgery-Comparison with Conventional Method. Asian Oncology Nursing, 2014, 14.4: 249-253

13. Liu $\mathrm{M}-\mathrm{Y}$, et al. Influence of preoperative peripheral parenteral nutrition with micronutrients after colorectal cancer patients. BioMed research international, 2015.

14. Gillis $C$, et al. Nutrition adequacy in enhanced recovery after surgery: a single academic center experience. Nutrition in Clinical Practice, 2015, 30.3: 414-419.

15. Melnyk M, Casey R G, Black P, \& Koupparis A J. Enhanced recovery after surgery (ERAS) protocols: Time to change practice? Canadian Urological Association Journal; 2011, 5(5), 342 .

16. Wykes K, Taylor K, Wilkinson SA. An investigation into the perioperative nutritional management of open colorectal surgery patients in major Australian hospitals: a comparison with the ERAS guidelines. Nutrition \& Dietetics, 2013, 70.3: 175-180.

17. Mcelroy LM.; Codner PA.; Brasel K J. A pilot study to explore the safety of perioperative postpyloric enteral nutrition. Nutrition in Clinical Practice, 2012, 27.6: 777-780.

18. Cestonaro T. The reality of the surgical fasting time in the era of the ERAS protocol. Nutricion hospitalaria, 2014, 29.2.

19. Di Muzio M, Rosa F, Moriconi A. Nutrition in dementia: a challenge for nurses. Progress in Nutrition 2018;20(1):5-11.

20. Di Muzio M, Cammilletti V, Petrelli E, Di Simone E. Hand hygiene in preventing nosocomial infections: a nursing research. Ann Ig. 2015 Mar-Apr;27(2):485-91. 